\author{
REA - Revista de estudos ambientais (Online) \\ v.22, n. 1, p.15-23, jan./jun. 2020
}

F U R B

\title{
OTTOCODIFICAÇÃO DE PEQUENAS BACIAS HIDROGRÁFICAS NA AMAZÔNIA
}

\section{Laila Rover Santana ${ }^{1}$, Claudio José Cavalcante Blanco² e Francisco Carlos Lira Pessoa ${ }^{3}$}

\begin{abstract}
Resumo: A literatura apresenta algumas definições muito subjetivas para classificar pequenas bacias hidrográficas em função da área de drenagem. Isso ocorre, talvez, pelo menor interesse nessas bacias em relação às grandes e médias bacias hidrográficas. A motivação do presente estudo é identificar essas bacias na Amazônia, utilizando o método de Otto Pfafstetter. O método foi aplicado em um bacias hidrográficas obtidas pelo Modelo Digital de Elevação Hidrograficamente Condicionado (MDEHC), que utilizou imagens do Shuttle Radar Topography Mission (SRTM), com resolução de $90 \mathrm{~m}$. A delimitação de pequenas bacias foi realizada partindo-se do nível 2 da ottocodificação disponibilizado pela Agência Nacional de Águas (ANA). Ao todo foram delimitadas 51.319 pequenas bacias, todas com áreas de drenagem menores ou iguais a $500 \mathrm{~km}^{2}$. Assim, para se identificar pequenas bacias hidrográficas na Amazônia através do método de ottocodificação, é necessário aplicar o método até o nível 9.
\end{abstract}

Palavras-chave: Área de drenagem. Modelo Digital de Elevação Hidrograficamente Condicionado. Amazônia.

\section{Introdução}

A bacia hidrográfica é uma das unidades territoriais mais adotadas nos estudos ambientais, sobretudo aos que se referem à gestão dos recursos hídricos. No território brasileiro, a Política Nacional de Recursos Hídricos (PNRH), instituída pela Lei no 9.433 (BRASIL,1997), define a bacia hidrográfica como unidade territorial básica para a gestão dos recursos hídricos que visa atender as demandas dos usos múltiplos da água, solucionar os conflitos entre usuários e meio ambiente, e proteger a utilização dos recursos da bacia a fim de alcançar o desenvolvimento sustentável da mesma (VOLLMER et al., 2018; KINGSFORD et al., 2017; ASMAMAW, 2015). Nesse contexto, definir a dimensão de uma pequena, média ou grande bacia hidrográfica é importante, pois o tamanho da bacia interfere diretamente nas atividades de gestão e planejamento dos recursos hídricos, uma vez que quanto maior a bacia maior serão os gastos para gerir adequadamente os seus recursos.

$\mathrm{Na}$ literatura são encontradas diversas definições para bacia hidrográfica, sendo que os autores adotam conceitos bem semelhantes, caracterizando-a, em geral, quanto a sua área de captação (LIU, 2015; WAGENER et al., 2007). Contudo, as

1E-mail: Santana1laila.santana@itec.ufpa.br

2E-mail: blanco@ufpa.br

UFPA - Rua Augusto Corrêa, 01, CEP. 66075-110, Belém-PA

${ }^{3}$ E-mail: fclpessoa@ufpa.br pequenas bacias hidrográficas são objeto de diversos estudos que buscam analisar seu comportamento hidrológico, bem como os fatores físicos, químicos e climáticos que podem influenciar no seu comportamento (KIJOWSKA-STRUGAŁA, 2019; JIANG et al., 2018; BELLOS; TSAKIRIS, 2016). O tamanho dessas bacias ainda é alvo de subjetividade, não havendo uma definição do valor de área de drenagem que limite a dimensão de uma bacia hidrográfica como sendo pequena (GOLDENFUM, 2003).

Buscando definir um valor de área para classificar as pequenas bacias hidrográficas na Amazônia, o estudo de Santana e Blanco (2020) utilizou um Modelo Linear Simples (MLS) para observar a linearidade entre os processos chuva-vazão. Segundo Blanco et al. (2013), essa linearidade é favorecida nessas bacias. Os resultados do estudo indicaram como classificação de pequenas bacias hidrográficas da Amazônia aquelas com áreas de drenagem menores que $500 \mathrm{~km}^{2}$.

O Conselho Nacional de Recursos Hídricos (CNRH) adota como codificação oficial das bacias hidrográficas brasileiras 0 sistema desenvolvido pelo engenheiro Otto Pfafstetter, conhecido como "Ottocodificação". A metodologia de codificação de Otto destaca-se de outros 
métodos de codificação devido à facilidade de implementação e interpretação (STEIN, 2018), sendo adotada em diversos estudos para delimitação e classificação de bacias hidrográficas (CURTIS et al., 2018; MULLER; ROLIM.; MARCUZZO, 2018; TRINDADESANTOS et al., 2018; PACHRI et al., 2013).

A codificação de Otto Pfafstetter, também chamada de "Ottocodificação", baseia-se na identificação do rio principal, e posteriormente, codificam-se suas bacias afluentes por área de contribuição. De acordo com a metodologia de Pfafstetter (1989), primeiro é identificado o rio principal da bacia a ser codificada. Em seguida, são separadas as quatro maiores bacias ao longo do rio principal, as quais recebem como código, em ordem de jusante para montante, os números pares 2, 4, 6 e 8. As bacias restantes, chamadas de interbacias, recebem como códigos os números ímpares 1, 3, 5, 7 e 9, também atribuídos de jusante para montante. O processo de subdivisão das bacias e das interbacias pode ser realizado quantas vezes for necessário em vários níveis, desde que o detalhamento da base cartográfica permita. Em cada novo nível, adiciona-se um número a direita no código da bacia codificada.

A metodologia de Otto também é de fácil integração com os Sistemas de Informações Geográficas (SIGs), que permitem a delimitação de bacias hidrográficas de forma automatizada, reduzindo o tempo de processamento dos dados e a probabilidade de erros (JÚNIOR et al., 2015). Diversos estudos foram realizados utilizando SIGs e a metodologia de Otto Pfafstetter (ROMANHOLI; FILHO, 2018; LEMOS et al., 2017; SCHRÖDER; OMRAN, A. F.; BASTIDAS, 2015). A delimitação através de ferramentas computacionais necessita de Modelos Digitais de Elevação (MDE), que armazenam valores de altitude do terreno (JUMBO et al., 2017). Contudo, esses modelos podem apresentar erros, que podem interferir no cálculo do fluxo das águas nas bacias a serem delimitadas (PAN; STIEGLITZ; MCKANE, 2012; PETROSELLI; ALVAREZ, 2012). Por isso, em estudos hidrológicos deve-se utilizar o Modelo Digital de Elevação Hidrograficamente Condicionado (MDEHC), pois este modelo possui como parâmetros básicos a direção do fluxo e a área de contribuição, respeitando o traçado da área mapeada.

Assim, com a finalidade de identificar essas pequenas bacias hidrográficas, este estudo tem como principal objetivo aplicar o método de Otto Pfafstetter às bacias da
Amazônia e identificar em que nível de ramificação são encontradas bacias com áreas de drenagem menores que $500 \mathrm{~km}^{2}$. Os rios da Amazônia atendem a diversas necessidades humanas, como saneamento, transporte fluvial, pesca, irrigação e produção de água potável e energia (WONGCHUIG et al., 2019), por isso se faz necessária uma adequada gestão e planejamento dos recursos hídricos dessa região, gerando a motivação de definição de pequenas bacias hidrográficas em função da área de drenagem. Uma vez essas bacias hidrográficas estando delimitadas e identificadas, elas poderão ser utilizadas em diversos estudos, pois mudanças nos regimes hidrológicos são mais sensíveis em pequenas bacias hidrográficas.

\section{Metodologia}

\section{1 Área de Estudo}

A Amazônia (Figura 1) possui uma área que corresponde a aproximadamente $59 \%$ do território brasileiro, abrangendo os Estados do Acre, Amapá, Amazonas, Pará, Rondônia, Roraima, Tocantins, Mato Grosso e parte do Estado do Maranhão (a oeste do meridiano $44^{\circ}$ ), totalizando uma área superior a 5 milhões de $\mathrm{km}^{2}$ (IBGE, 2014).

\subsection{Aplicação da Ottocodificação para determinação de pequenas bacias hidrográficas}

A área de drenagem das bacias foi definida com base no estudo de Santana e Blanco (2020), que utilizou o Modelo Linear Simples (MLS) chuva-vazão (BLANCO et al., 2005) para classificar as bacias hidrográficas da Amazônia em função da área de drenagem. As hipóteses de linearidade e invariabilidade no tempo do modelo permitem estabelecer uma relação de causa e efeito entre os dados de chuva e vazão, e essa relação é observada em pequenas bacias. $O$ MLS foi testado em 26 bacias hidrográficas da Amazônia com áreas de drenagem variando entre 32,7 e $1810 \mathrm{~km}^{2}$. Os resultados indicaram que, para bacias maiores que 500 $\mathrm{km}^{2}$, o desempenho do modelo foi reduzido, demonstrando que a hipótese de linearidade do modelo foi quebrada, estabelecendo um ponto de corte referente a pequenas bacias hidrográficas (SANTANA; BLANCO, 2020). 
v.22, n. 1, p.15-23, jan./jun. 2020

Figura 1 - Mapa do Brasil com destaque para a localização da Amazônia

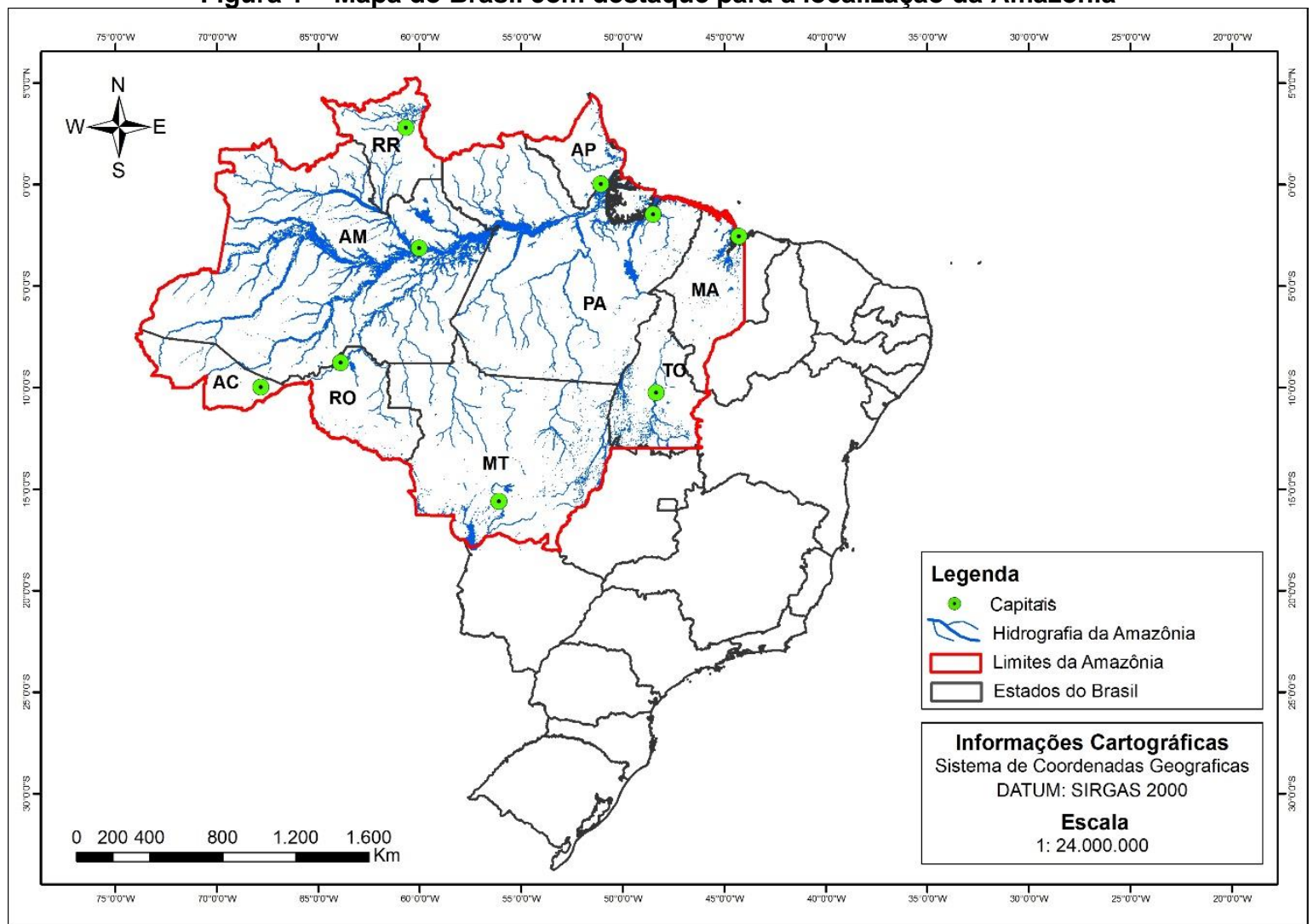

Fonte: IBGE (2014), IBAMA (2010).

Assim, para efeito da aplicação do método de Otto, a codificação foi realizada até o nível em que foram encontradas bacias hidrográficas com áreas menores ou iguais a $500 \mathrm{~km}^{2}$.

O sistema proposto por Otto Pfafstetter baseia-se na topografia da área drenada e na topologia da rede de drenagem. Nesse caso, o uso de MDEHC torna-se indispensável na ottocodificação, pois permite a delimitação das ottobacias com maior precisão. Para obtenção do MDEHC, primeiramente, foram selecionadas as imagens Shuttle Radar Topography Mission (SRTM), dos Estados que compõem a Amazônia, disponibilizadas no site da Empresa Brasileira de Pesquisa Agropecuária (EMBRAPA), com resolução espacial de 90 metros (MIRANDA et al., 2005).

Para o tratamento das imagens SRTM e obtenção do MDEHC, foi utilizado o software ArcGIS® 10.2, do Environmental Systems Research Institute (ESRI, 2013). O conjunto de extensões utilizado para o tratamento das imagens, extração da hidrografia e delimitação das sub-bacias foi o do módulo Arc HydroTools do ArcToolbox®.
Diversos estudos vêm sendo realizados com - Archydro para delimitação de bacias hidrográficas (IMRAN et al., 2019; CASSETTARI; DE QUEIROZ, 2019; TEFERA, 2017; OLIVEIRA; ANTÔNIO, 2015; LOPES et al., 2011), uma vez que esta ferramenta foi criada para resumir dados geoespaciais e temporais relacionados aos recursos hídricos para análises hidrológicas (JÚNIOR et al., 2015). As etapas para obtenção do MDHEC e para delimitação das bacias hidrográficas, utilizando o Arc Hydro Tools estão descritas no esquema metodológico da Figura 2. Nessa figura foi mantida a nomenclatura em língua inglesa inerente ao software $\operatorname{ArCGIS} \circledast 10.2$.

A primeira etapa na geração do MDEHC consiste no preenchimento de depressões espúrias, que segundo Elesbon et al. (2011), são altitudes equivocadas devido ao processo de interpolação ou obtenção da imagem por satélite. A função FILL SINKS foi utilizada para corrigir essas falhas. A etapa seguinte foi determinar a direção do fluxo de água na rede de drenagem, sendo obtida através da função FLOW DIRECTION. Esta função gera uma grade regular definindo as direções de fluxo, 
tendo como base a linha de maior declividade do terreno (SOBRINHO et al., 2010). A partir da direção do fluxo, é gerado o fluxo acumulado do MDE através da função FLOW
ACCUMULATION. Em seguida foram aplicadas as funções STREAM DEFINITION e STREAM SEGMENTATION para definir a quantidade de feições de drenagens geradas.

Figura 2 - Metodologia de delimitação de bacias hidrográficas utilizando a extensão Arc Hydro Tools

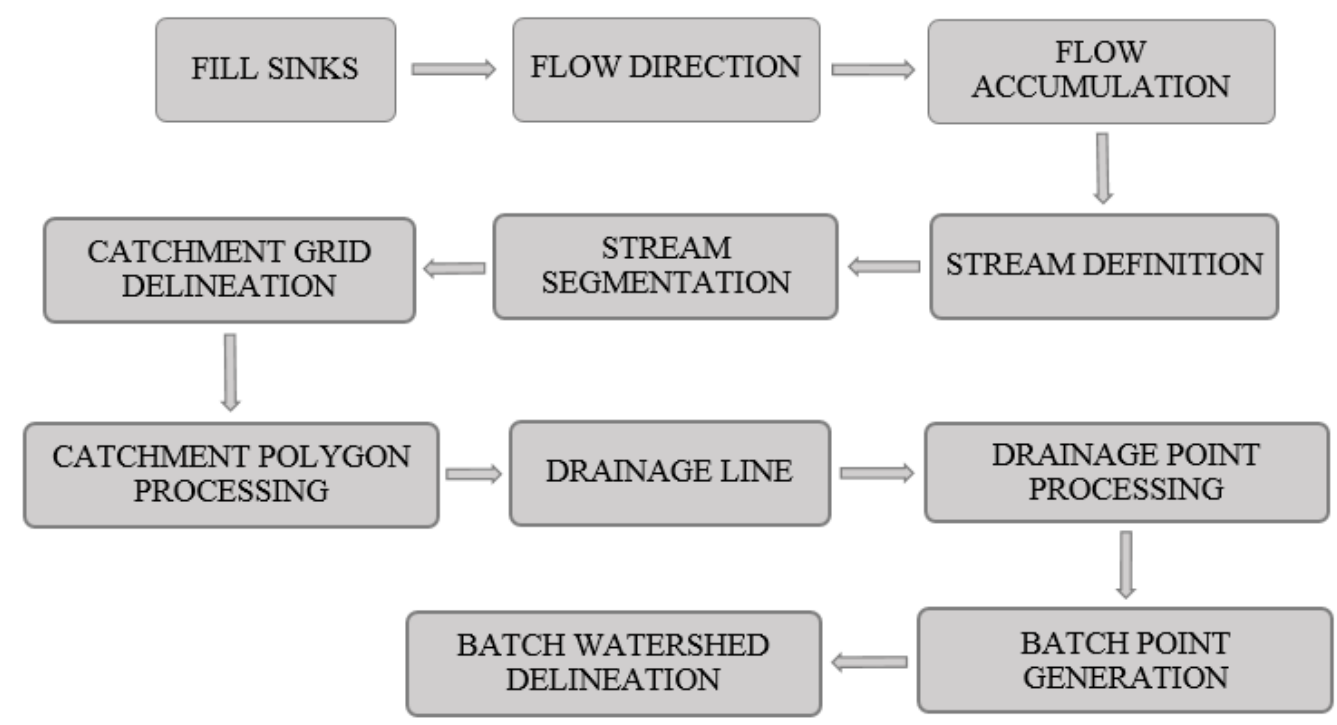

Fonte: Autores (2020)

Com a definição da segmentação do fluxo, foram aplicadas as funções CATCHMENT GRID DELINEATION e CATCHMENT POLYGON PROCESSING. Essas ferramentas permitem criar os polígonos de bacias hidrográficas a partir das redes de captação, e pela conversão das redes de ligação de fluxo em feições de linha de drenagem (TEFERA, 2017). Posteriormente, são aplicadas as funções DRAINAGE LINE e DRAINAGE POINT PROCESSING, que convertem os dados raster em formato vetorial. Por fim, a delimitação das bacias ocorre pela função BATCH WATERSHED DELINEATION, que utiliza os arquivos de linhas de fluxo, fluxo acumulado, linhas de drenagem e delimitação preliminar das bacias geradas anteriormente (TEFERA, 2017). Porém, primeiro é necessário criar o ponto do exutório da bacia a partir da ferramenta BATCH POINT GENERATION, e em seguida delimita-se a bacia desejada.

De posse do MDEHC da área de estudo, a metodologia de Otto Pfafstetter foi aplicada em todas as bacias hidrográficas geradas pelo modelo. Ressalta-se que para este estudo, a codificação foi realizada a partir de uma drenagem numérica gerada pela acumulação de 50 células do MDEHC. Esse valor foi definido a partir de comparações realizadas com a base hidrográfica ottocodificada da Agência Nacional de Águas (ANA), buscando-se aproximar, ao máximo, a drenagem gerada a partir do MDEHC com a drenagem da ANA.

O sistema de informações da ANA disponibiliza, para o Brasil, as bacias ottocodificadas até o nível 6 (ANA, 2012). Nota-se que 0 primeiro nível dividiu a Amazônia em 6 grandes regiões hidrográficas (Figura $3 \mathrm{~A}$ ), sendo a de número 4 , a bacia hidrográfica do Amazonas, a maior de todas, com uma área de aproximadamente $3.900 .000 \mathrm{~km}^{2}$. Ressalta-se que esse valor engloba apenas os limites brasileiros. No nível 2 da ottocodificação, as 6 bacias do nível 1 foram divididas em 32 ottobacias (Figura 3B). Vale lembrar que, ambos os mapas, apresentam os limites da Amazônia, por isso nem todas as ottobacias das regiões hidrográficas Costeira do Atlântico Norte e Costeira do Atlântico do Sul, e da Bacia hidrográfica do Paraná estão representadas, uma vez que se encontram fora dessa delimitação.

Para este estudo, a ottocodificação foi aplicada a partir do nível 2 fornecida pelo banco de dados da ANA (Figuras 3A e 3B). 
Figura 3 - Ottocodificação da ANA aplicada às bacias hidrográficas da Amazônia, A) nível 1, B) nível 2

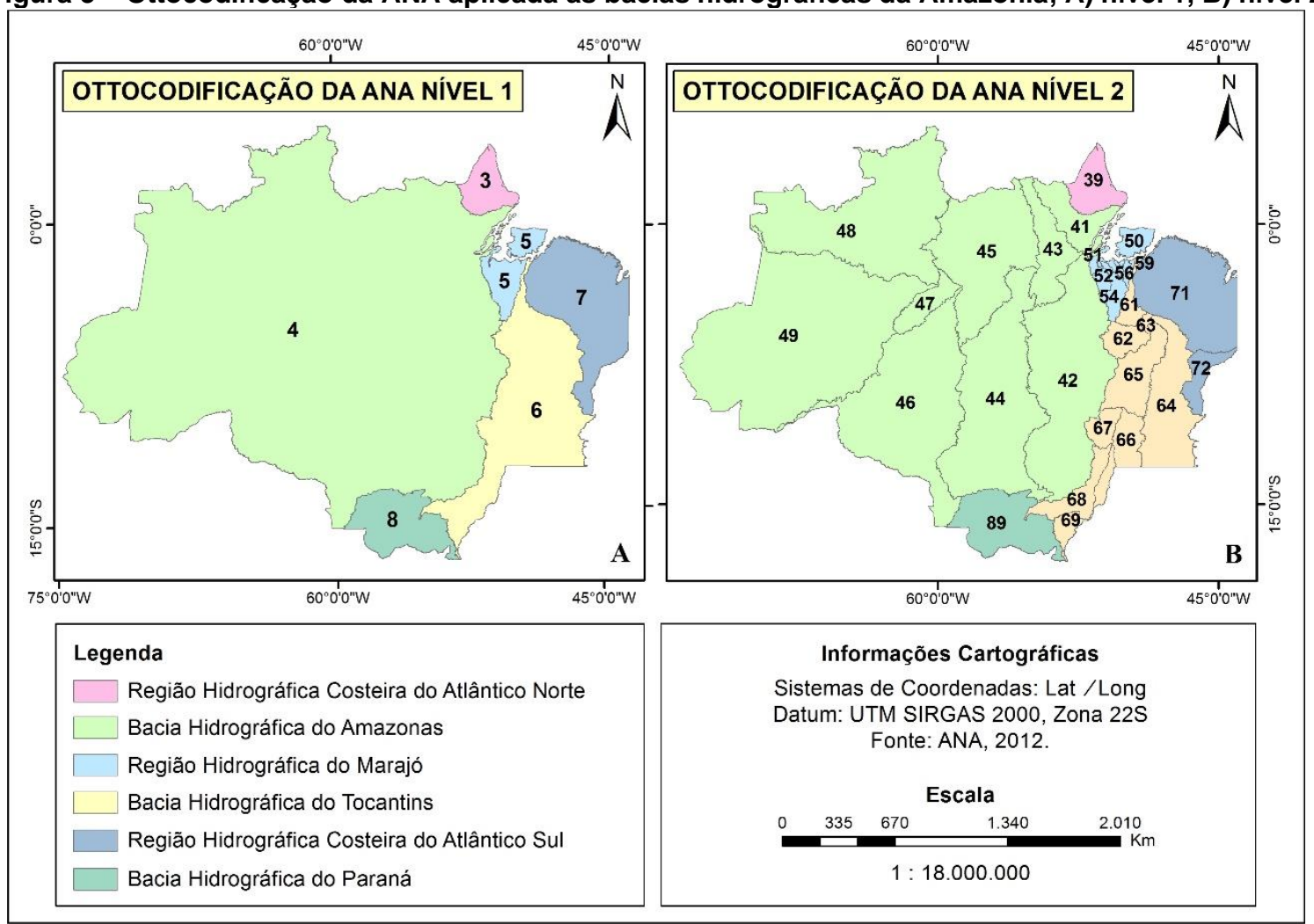

Fonte: ANA (2012)

\section{Resultados}

$\mathrm{Na}$ Figura 4 são apresentados os níveis da Ottocodificação aplicada às bacias hidrográficas da Amazônia. Nota-se que os níveis 3 e 4 da ottocodificação das bacias da Amazônia apresentaram o menor número de pequenas bacias, 71 e 1.004 ottobacias, respectivamente, com áreas de até $500 \mathrm{~km}^{2}$. Contudo, nota-se que no nível 4, a Região Hidrográfica do Marajó (Figura $3 A$ ) já apresenta um número significativo de pequenas bacias delimitadas. Esse resultado pode ser explicado pela presença de bacias com áreas menores nessa região da Amazônia (Figura 3B).

No nível 5 foram delimitadas 9.886 bacias, sendo que dessas, 7.640 possuem áreas $\leq 500 \mathrm{~km}^{2}$, definidas como pequenas bacias hidrográficas. Já no nível 6 , observase que houve um aumento expressivo no número de pequenas bacias identificadas pelo método da ottocodificação, totalizando 28.722 bacias classificadas como pequenas. Nota-se ainda que na região da bacia hidrográfica do Amazonas (Figura $3 \mathrm{~A}$ ) a área em azul, onde as bacias ottocodificadas ainda possuem áreas de drenagem maiores que
$500 \mathrm{~km}^{2}$, é maior que nas demais regiões. Isto porque nessa área as bacias são maiores, sendo necessário realizar a subdivisão em mais níveis para se identificar pequenas bacias hidrográficas.

Nos níveis 7 e 8 foram delimitadas 46.411 e 50.901 ottobacias, respectivamente, sendo que o número de pequenas bacias hidrográficas identificadas foi de 46.061 no nível 7 e 50.882 no nível 8 da ottocodificação. Por fim, no nível 9 foram delimitadas 51.319 ottobacias, as quais possuem áreas de drenagem $\leq 500 \mathrm{~km}^{2}$, ou seja, todas classificadas como pequenas bacias hidrográficas. A delimitação dessas bacias utilizando ferramentas de SIG possibilitou a construção de uma base de dados de bacias hidrográficas da Amazônia com maior precisão e em menor tempo. A ottocodificação também permite a busca e a identificação, de maneira mais rápida, por pequenas bacias hidrográficas, que são unidades ambientais comumente utilizadas no planejamento e gestão dos recursos hídricos de uma região. 
v.22, n. 1, p.15-23, jan./jun. 2020

Figura 4 - Níveis da Ottocodificação aplicada às bacias hidrográficas da Amazônia

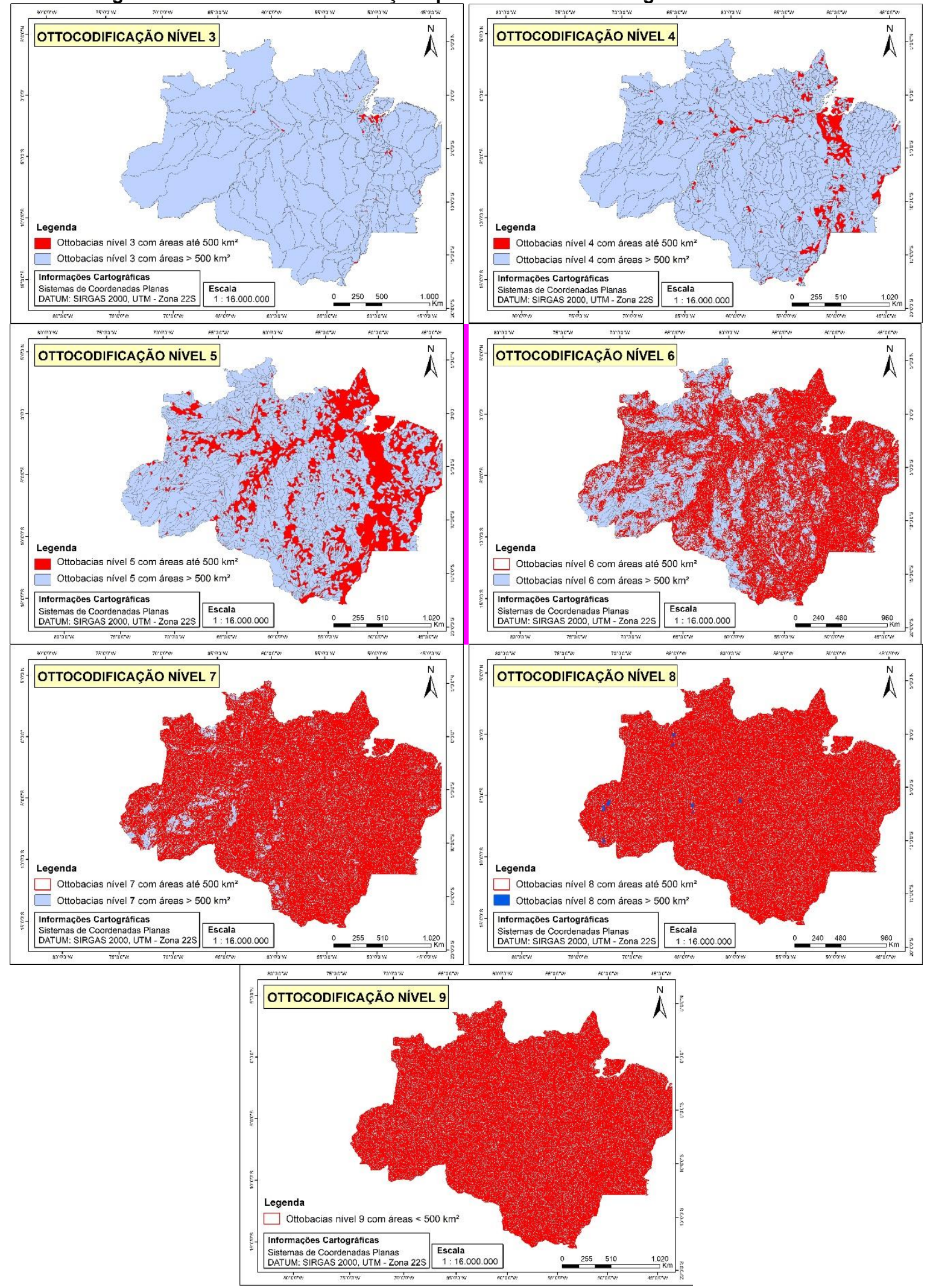

Fonte: Autores (2020) 


\section{Conclusões}

O método de Otto mostrou-se eficaz na delimitação das pequenas hidrográficas bacias da Amazônia. Assim como o MDEHC obtido das imagens SRTM com resolução espacial de 90 metros mostrou-se eficaz para delimitação dessas unidades hidrográficas. Por tomar como base a topografia da área drenada e a topologia da rede de drenagem, a metodologia de Otto Pfafstetter possibilita a delimitação de bacias hidrográficas com maior precisão, e a integração com SIGs permite a aplicação automática do método. Com o método de ottocodificação associado as ferramentas de geoprocessamento foram delimitadas 51.319 ottobacias, todas com áreas de drenagem menores que $500 \mathrm{~km}^{2}$. Nesse contexto, os resultados da ottocodificação indicam que, para se obter apenas pequenas bacias hidrográficas na Amazônia, é necessário aplicar a metodologia de Otto Pfafstetter até o nível 9.

\title{
5 Pfafstetter Coding System of Small Watershed in the Amazon
}

\begin{abstract}
The literature presents some very subjective definitions to take it account drainage area in order to consider watersheds as a small one. This fact is perhaps related to the greater interest on large and medium watersheds than in the small ones. The aim of this study is to identify these basins in the Amazon using the Otto Pfafstetter method. The method was applied in watersheds obtained by Conditioning Hydrographic Digital Elevation Model (CHDEM), which used $90 \mathrm{~m}$ resolution Shuttle Radar Topography Mission (SRTM) images. The small watersheds delimitation was carried out starting from level 2 of Pfafstetter Coding provided by the National Water Agency (ANA). In total, 51,319 small watersheds were defined, all with drainage areas smaller or equal to $500 \mathrm{~km}^{2}$. Thus, to identify small watersheds in the Amazon using the Pfafstetter Coding, it is necessary to apply the method up to level 9.
\end{abstract}

Keywords: Drainage areas, Conditioning Hydrographic Digital Elevation Model, Amazon.

\section{Referências}

ANA. AGÊNCIA NACIONAL DE ÁGUAS E SANEAMENTO BÁSICO (Brasil). Dados abertos da ANA (2012). Disponível em: https://metadados.ana.gov.br/geonetwork/srv/pt/m ain.home?uuid=b228d007-6d68-46e5-b30da1e191b2b21f. Acesso em: out. 2019.

ASMAMAW, D. K. A critical review of integrated river basin management in the upper Blue Nile river basin: the case of Ethiopia. International Journal of River Basin Management 13, 429-442, 2015.

BELLOS, V.; TSAKIRIS, G. A hybrid method for flood simulation in small catchments combining hydrodynamic and hydrological techniques. Journal of Hydrology, 540, 331-339, 2016.

BLANCO, C. J. C.; SANTOS, S. S. M.; QUINTAS, M. C.; VINAGRE, M. V. A.; MESQUITA, A. L. A. Contribution to hydrological modelling of small Amazonian catchments: application of rainfallrunoff models to simulate flow duration curves. Hydrological Sciences Journal, 58(7), 14231433, 2013.

BLANCO, C. J.; SECRETAN, Y.; FAVRE, A. C.; SLIVITZKY, M. Modèle pluie-débit pour la simulation de courbes de débits classés sur des petits bassins non jaugés de l'Amazonie. Canadian Journal of Civil Engineering, v.32, p.803-811, 2005.

BRASIL. Lei no 9.433, de 8 de janeiro de 1997. Institui a Política Nacional de Recursos Hídricos, cria o Sistema Nacional de Gerenciamento de Recursos Hídricos, regulamenta o inciso XIX do art. 21 da Constituição Federal, e altera 0 art. $1^{\circ}$ da Lei $\mathrm{n}^{\circ}$ 8.001, de 13 de março de 1990, que modificou a Lei $n^{\circ} \mathbf{7 . 9 9 0}$, de 28 de dezembro de 1989. Diário Oficial da República Federativa do Brasil, Brasília, DF 09/01/1997, P. 470. Disponível em: <planalto.gov.br/ccivil_03/leis/19433.htm>. Acesso em: 10/09/2019.

CASSETTARI, G. A.; DE QUEIROZ, T. M. Morphometric characterization of the hydrographic basin of Jauquara river in the transition between the Cerrado and Amazon biomes in Mato GrossoBrazil. Floresta, 49(2), 325-334, 2019.

CURTIS, S.; CRAWFORD, T.; RAHMAN, M.; PAUL, B.; MIAH, M.; ISLAM, M.; PATEL, M. A Hydroclimatological Analysis of Precipitation in the Ganges-Brahmaputra-Meghna Basin. Water, 10(10), 1359, 2018. River

ELESBON, A. A. A.; GUEDES, H. A. S.; SILVA, D. D. D.; CASTRO E OLIVEIRA, I. D. Uso de dados SRTM e plataforma SIG na caracterização morfométrica da bacia hidrográfica do Braço Norte 
do Rio São Mateus - Brasil. Rem: Revista Escola de Minas, Ouro Preto, v. 64, n. 3, p. 281-288, 2011.

INSTITUTO BRASILEIRO DE GEOGRAFIA E ESTATÍSTICA - IBGE. Amazônia Legal (2014). Disponível em https://www.ibge.gov.br/geociencias/organizacaodo-territorio/estrutura-territorial/15819-amazonialegal.html?edicao=16194\&t=0-que-e. Acesso em: mar. 2019.

ENVIRONMENTAL SYSTEMS RESEARCH INSTITUTE - ESRI. ArcGIS $\mathbf{1 0 . 2}$ for Desktop. Versão 10.2.0.3348. ESRI, 2013.

GOLDENFUM, J. A. Pequenas bacias hidrográficas: conceitos básicos. In: PAIVA, E. M. C. D. de; PAIVA. J. B. D. de (Org.). Hidrologia aplicada à gestão de pequenas bacias hidrográficas. Porto Alegre: ABRH, p.3-13, 2003.

IMRAN, R. M.; REHMAN, A.; KHAN, M. M.; RAHAT, M.; JAMIL, U. A.; MAHMOOD, R. S.;MAHMOOD, S. A.; EHSAN, R. M. Delineation of Drainage Network and Estimation of Total Discharge using Digital Elevation Model (DEM). Science and Technology, 1(02), p. 50-61, 2019.

JIANG, H.; LIU, W.; XU, Z.; ZHOU, X.; ZHENG, Z.; ZHAO, T.; ZHOU, L.; ZHANG, X.; XU, Y.; LIU, T. Chemical weathering of small catchments on the Southeastern Tibetan Plateau I: Water sources, solute sources and weathering rates. Chemical Geology, 500, p. 159-174, 2018.

JUMBO, F.; ZEA, M.; LOJA, N.; MOROCHO, R.; CARTUCHE, J.; LOJÁN, E. Delimitation and Codification of Hydrographic Units Through the Use of Geographic Information Systems. In International Conference on Technology Trends, p. 185-198. Springer, Cham, 2017.

JÚNIOR, C. B.; DE SOUZA, F. A. O.; MAUAD, F. F. GIS Automation for Spatialization of Water Availability. Anuário do Instituto de Geociências, 38 (2), 2015.

KIJOWSKA-STRUGA $Ł A, M$. Sediment variability in a small catchment of the Polish Western Carpathians during transition from centrally planned to free-market economics. Geomorphology, 325, p. 119-129, 2019.

KINGSFORD, R. T.; BINO, G.; PORTER, J. L. Continental impacts of water development on waterbirds, contrasting two Australian river basins: global implications for sustainable water use. Global ChangeBiology 23, p. 4958-4969, 2017.

LEMOS, C. E. F.; DE SOUZA VIEIRA, M. I.; FERRAZ, R. R. Compartimentação hidrográfica da bacia de drenagem do Rio Branco, estado de Roraima, Brasil por meio da base hidrográfica ottocodificada. ActaGeográfica, 11(26), p. 35-53, 2017.

LIU, N. Emergence, concept, and understanding of Pan-River-Basin (PRB). International Soil and Water Conservation Research, v. 3, p. 253-260, 2015.

LOPES, F.B.; ANDRADE, E.M.; TEIXEIRA, A.S.; CAITANO, R.F.; CHAVES, L.C.G. Uso de geoprocessamento na estimativa de perda de solo em microbacia hidrográfica do semiárido brasileiro. Revista Agro@mbiente on-line, 5(2): p. 88-96, 2011.

MIRANDA, E. E. de; (Coord.). Brasil em Relevo. Campinas: Embrapa Monitoramento por Satélite, 2005. Disponível em: http://www.relevobr.cnpm.embrapa.br. Acesso em: out. 2019.

MULLER, Y. T.; ROLIM, F. E.; MARCUZZO, F. F. $N$. Ottocodificação e análise altimétrica e da precipitação pluviométrica da bacia hidrográfica do rio Pelotas na divisa entre o Rio Grande do Sul e Santa Catarina. Geographia Meridionalis, v. 04, n. 02, p. 227-245, 2018.

OLIVEIRA, B. R.; ANTÔNIO, G. B. Caracterização da bacia do Maxixe com auxílio de Sistemas de Informações Geográficas. Electronic Journal of Management, Education and Environmental Technology (REGET), 19(3), p. 761-782, 2015.

PACHRI, H.; MITANI, Y.; IKEMI, H.; DJAMALUDDIN, I.; MORITA, A. Development of water management modeling by using GIS in Chirchik river basin, Uzbekistan. Procedia Earth and Planetary Science, 6, p. 169-176, 2013.

PAN, F., STIEGLITZ, M., MCKANE, R. An algorithm for treating flat areas and depressions in digital elevation models using linear interpolation. Water Resources Research, v. 48, n. 6, 2012.

Petroselli, A., Alvarez, A., F. The flat-Area issue in digital elevation models and its consequences for rainfall-Runoff modelling. GIScience \& Remote Sensing, 49 (5), p. $711-$ 734, 2012.

PFAFSTETTER, O. Classificação de bacias hidrográficas - metodologia de codificação. Rio de Janeiro, RJ: DNOS, 19p, 1989.

ROMANHOLI, M. P.; FILHO, A. P. de Q. Base hidrográfica ottocodificada na escala 1:25.000: exemplo da bacia do córrego Itapiranga (SP). Caminhos de Geografia, v.19, n.68, p.46-60, 2018.

SANTANA, L. R.; BLANCO, C. J. C. Contribution to the classification of small catchments according to the drainage area. International Journal of River Basin Management, p.1-25, 2020. 
SCHRÖDER, D.; OMRAN, A. F.; BASTIDAS, M. R. R. Automated Geoprocessing Workflow for Watershed Delineation and Classification for Flash Flood Assessment. International Journal of Geoinformatics, 11 (4), 2015.

SOBRINHO, A. T.; OLIVEIRA, P. T. S. D.; RODRIGUES, D. B. B.; AYRES, F. M. Delimitação automática de bacias hidrográficas utilizando dados SRTM. Engenharia

Agrícola, Jaboticabal, v. 30, n. 1, p.46-57, 2010.

STEIN, J. L. An enhanced Pfafstetter catchment reference system. Water Resources Research, 54(12), 9951-9963, 2018.

TEFERA, A. H. Characterization of Beles River Basin of Blue Nile sub-Basin in North-Western Ethiopia using Arc-Hydro tools in ArcGIS. International Journal of Water Resources and Environmental Engineering, 9(5), p. 113120, 2017.

TRINDADE-SANTOS, I.; EDUARDO, A. A.; MOYES, F.; MARTINEZ, P. A.; MAGURRAN, A. E.; GOUVEIA, S. F. Simulating shifts in taxonomic and functional $\beta$-diversity of ray-finned fishes: Probing the Mariana disaster. Perspectives in Ecology and Conservation, v. 16 (4), p.186-192, 2018.

VOLLMER, D.; SHAAD, I.; SOUTER, N.J.; FARRELL, T.; DUDGEON, D.; SULLIVAN, C. A.; FAUCONNIER, I.; MACDONALD, G.; MCCARTNEY, M. P.; POWER, A.G.; MCNALLY, A.; ANDELMAN, S. J.; CAPON, T.; DEVINENI, N.; APIRUMANEKUL, C.; NAM NG, C.; SHAW, M.R.; WANG, R.Y.; REGAN, H.M.Integrating the social, hydrological and ecological dimensions of freshwaterhealth: the Freshwater Health Index. Science of the Total Environment, 627, 304-313, 2018.

WAGENER, T.; SIVAPALAN, M.; TROCH, P.; WOODS, R. Catchment Classification and Hydrologic Similarity. Geography Compass, v.1, (4), p. 901-931, 2007.

WONGCHUIG, S. C., DE PAIVA, R. C. D., SIQUEIRA, V., \& COLLISCHONN, W. Hydrological reanalysis across the 20th century: A case study of the Amazon Basin. Journal of Hydrology, 570, 755-773, 2019. 